\title{
Effects of Out-of-plane Brace-to-chord Angle on Multiplane CHS X-joints Behavior Under Brace Compression
}

\author{
Bida Zhao', Ke Li ${ }^{1}$, Chengqing Liư ${ }^{2,3^{*}}$, Dengjia Fang ${ }^{2}$, Jianguo $\mathrm{Wu}^{1}$ \\ ${ }^{1}$ College of Civil Engineering and Architecture, \\ Zhejiang University of Technology, Hangzhou 310014, China \\ 2 School of Civil Engineering, \\ Southwest Jiaotong University, Chengdu 610031, China, \\ ${ }^{3}$ Key Laboratory of High-speed Railway Engineering of Ministry of Education, Chengdu 610031, China \\ *Corresponding author, e-mail: Icqjd@swjtu.edu.cn
}

Received: 16 January 2019, Accepted: 30 May 2019, Published online: 08 August 2019

\begin{abstract}
Multiplanar CHS X-joints, different from the common uniplanar CHS X-joints, usually with a relative small out-of-plane brace-to-chord angle (OPBCA) for appealing architectural appearance in the single layered lattice structures. In order to study the effects of OPBCA on the static behavior of circular hollow section (CHS) X-joints under brace axial compression, experimental tests and numerical parametric study on the ultimate capacity and load transfer pattern of the CHS X-joints were carried out. The numerical analysis results had good consistent with experimental tests in terms of the capacity and fail mode of the X-joints. OPBCA changes the load transfer pattern to more load at the up saddle point from the same load at the up and bottom saddles in uniplanar X-joints, and more obvious for the X-joints with lager OPBCA. OPBCA is also unfavorable to the capacity, especially the X-joints with relative large braceto-chord diameter ratio and in-plane brace-to-chord angle. Then an equation considering the OPBCA influence factor, extended the capacity prediction formulae of uniplanar X-joints in the current specifications to the multiplanar X-joints, is also established; and the equation has been validated favorably.
\end{abstract}

Keywords

CHS X-joints, out-of-plane brace-to-chord angle (OPBCA), load transfer pattern, ultimate capacity, OPBCA influence factor

\section{Introduction}

Unstiffened tubular joints, brace and chord tube members are often treated by directly welding the braces to the surface of the chords, have been widely used in engineering structures $[1,2]$ due to fabricate simple, cost-effective and aesthetically appealing. However, their ultimate capacity is low relatively. Therefore, the existing studies of tubular joints focused mainly on the ultimate static capacity and appropriate strengthening techniques. Based on the plastic load concept for limit analysis of pressure vessel proposed by Gerdeen [3], Choo et al. [4] presents a new approach in the definition of joint strength for thick-walled circular hollow section (CHS) unstiffened X-joint under brace axial loading, then the effects of chord stresses on the static strength of the X-joint were also studied by Choo et al. [5]. Fung and Soh et al. [6-7] investigated the ultimate capacity of completely overlapped CHS connections with the lap brace under axial compression. Feng and Young [8] presented theoretical investigation on cold-formed stainless steel square and rectangular hollow section (SHS and RHS) $\mathrm{T}$ - and X-joints under brace compression. Iskander et al. [9] carried out experimental tests and finite element (FE) studied on the through-bolts strengthened CHS T-joints under braces compression, and it was found that the ultimate capacity is increased by $35 \%$ when one through bolt is used. Zhu et al. [10, 11] performed experiment studied on the external rings strengthened CHS T- and $\mathrm{X}$-joints under braces compression. Fu et al. [12] performed experiments and finite element (FE) studied on the CHS gap K-joints strengthened with Carbon Fiber Reinforced Polymer (CFRP) sheets, the results revealed that the proposed technique of CFRP installation was efficient to promote performance of the K-joints [13]. Chen et al. [14] performed experimental and FE studied on the static behavior of double-skin CHS X-joints under axial compression, and 
proposed the corresponding design equations for the double-skin X-joints. Nassiraei et al. [15] performed FE analyzed to study the effects of doubler plate size and connections geometry on the ultimate strength, initial stiffness, and failure modes in CHS T- and Y-joints reinforced with doubler plate subjected axially tensile load [16]; and the results indicated that the doubler plate can significantly increase the initial stiffness, ultimate capacity, and considerably improve failure modes. Lan et al. [17] carried out numerical and theoretical studies on static strength of internally ring-stiffened CHS DT-joints subjected to brace axial compression or tension, and proposed equations for predicting the capacity of the DT-joints.

In practice, unstiffened CHS X-joints is one of common configurations connecting structural CHS members in single layered lattice steel tubular structures, which have become a popular choice for large-span roof systems. Unlike the previously studied uniplanar CHS X-joints, these $\mathrm{X}$-joints usual with out-of-plane brace-to-chord angle (OPBCA) for appealing architectural appearance, named multiplane CHS X-joints. The multiplanar X-joints are also different to the TT-joint mentioned in the specification of EN 1993-1-8:2005 [18] and Chinese standard GB 50017-2017 [19], both are usually applied to the single layered lattice structures and the spatial tube truss structures, respectively. Therefore, the TT-joints often has a relative large OPBCA, and the main internal force of these components, at the end of every members (brace), is axial forces; while the multiplanar $\mathrm{X}$-joints often has a relative small OPBCA, and the main internal force at the end of brace contains axial force, out-of-plane moment and in-plane moment. Moreover, two braces of the TT-joints usually are subjected different axial forces; however, two braces of the multiplanar X-joints have the same section size and the same value of internal forces (including moments and axial force). A typical engineering application of the multiplanar X-joints is shown in Fig. 1, where the OPBCA are ranging from $0^{\circ}$ to $20^{\circ}$.

The paper aims to study the effects of OPBCA on the static behavior of CHS X-joints subjected brace axial compression. An experiment with CHS X-joint under brace axial compression is first performed to verify the numerical simulation method using the package ABAQUS, then, FE parametric analysis is performed to investigate how OPBCA can influence the ultimate capacity and principle of mechanical transmission of the X- joint by comparing the results from multiplanar X-joints and corresponding uniplanar $\mathrm{X}$-joints. Moreover, extended the formula

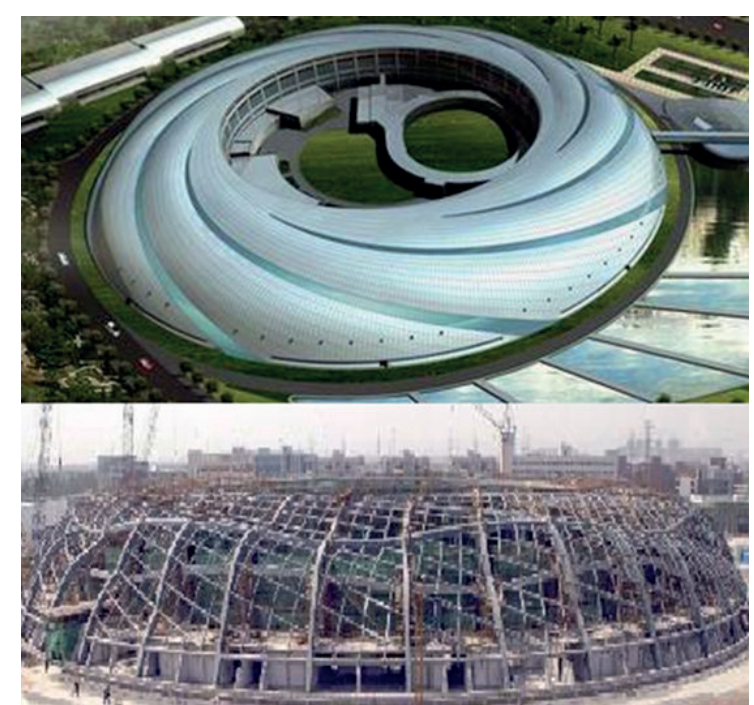

(a) Aerial view and site construction

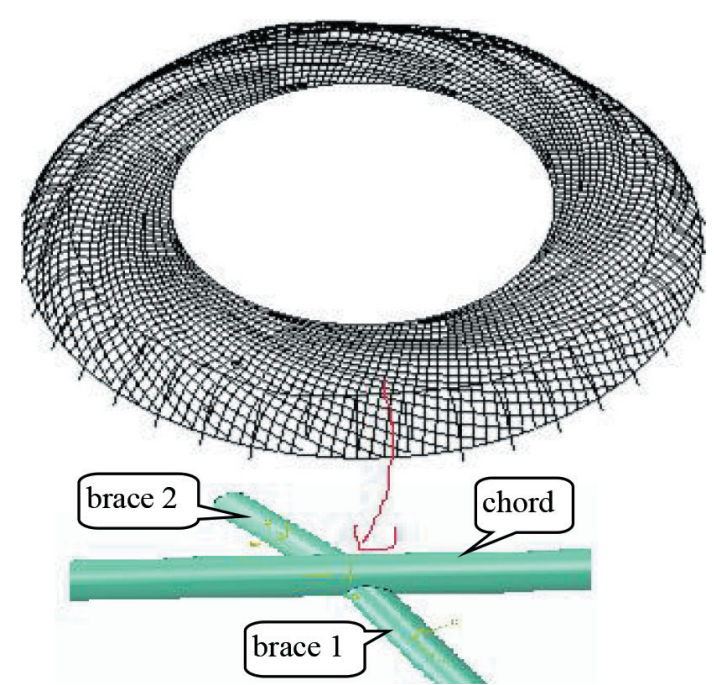

(b) Structure schemes and X-joint details

Fig. 1 Multiplanar CHS X-joints in practice

(in current specification $[18,19])$ that can not only be applied to the capacity of the uniplanar CHS X-joints, but also to the multiplanar CHS X-joints by multiplying OPBCA influence factor, which is determined by $\mathrm{FE}$ parameter analysis results and multiple regression analysis. Research results of this paper will provide technical support to promote the application of unstiffened CHS joints in the single-layer latticed structures.

\section{General FE model of multiplanar CHS X-joints and experiment verification}

\subsection{Geometric parameters and general FE model for the multiplanar CHS X-joints}

The configuration of multiplanar unstiffened CHS X-joints and the corresponding geometric parameters was illustrated in Fig. 2. Where, the two braces was of the same 


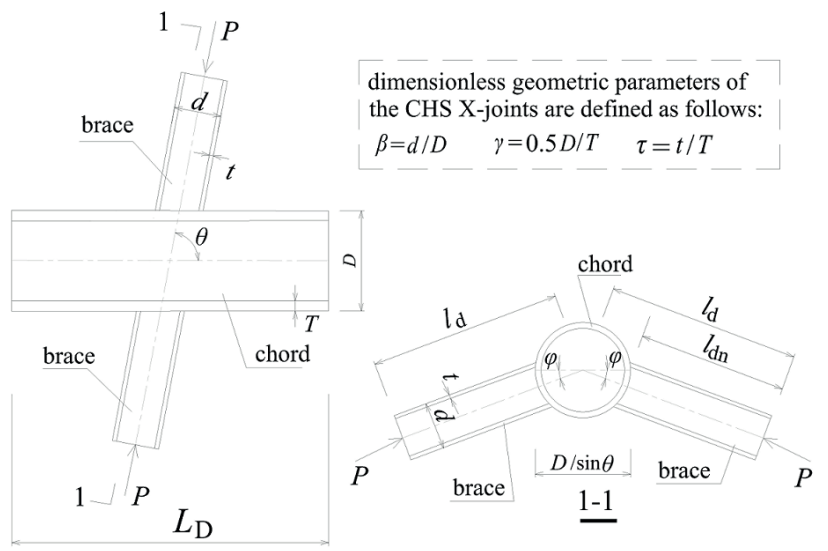

Fig. 2 Multiplanar CHS X-joints under brace compression

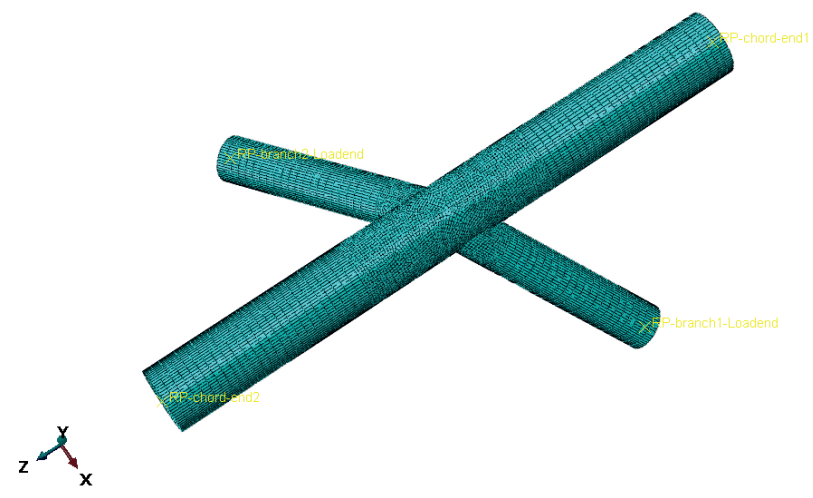

Fig. 3 General FE model with mesh layout

dimensions and subjected same compression $P$, and the in-plane brace-to-chord angle $\theta$ and the out-of-plane braceto-chord angle $\varphi$ of the two braces was also the same as shown in the figure, the geometrics of $\beta, \gamma$ and $\tau$ indicate the brace-to-chord diameter ratio, the chord radius to the chord wall thickness ratio and brace-to-chord wall thickness ratio, respectively.

In the general finite element (FE) model for the parameters study, the chord length ratio $\left(L_{D} / D\right)$ was 12 , and brace length ratio $\left(l_{d} / d\right)$ was 5 to reduce the effects of the boundary conditions on joint zone. The fixed boundary was applied to the two chord ends. The motion of every node on the free end section of brace were constrained together with the centroid point of the section (named control point) by Kinematic coupling constraint [20], then the displacement control was applied to the control point to simulate the brace axial compression. As for element meshing, eight-node reduced integration shell elements (S8R in ABAQUS [20]) were used in the FE models. These refined meshes with mesh size of chord thickness $(T)$ were used in the brace-to-chord intersection region in order to more accurately capture the stress or strain distributions, and the other regions of the joints adopted rectangular mesh sizes with length and width are $3 T$ and $T$ respectively. The typical FE mesh detail of a multiplanar CHS X-joint was shown in Fig. 3.

In the FE models for parameters analyses, the geometric nonlinear behavior was considered during the analyses by using the 'NLGEOM' option in ABAQUS [20], and material nonlinearity was accounted for in the analyses through a classical plasticity model based on the Von Mises yield criterion with the isotropic hardening rule. A bilinear hardening was used to describe the stress-strain relationship of steel material, with the elasticity modulus $(E)$ taken as $206 \mathrm{GPa}$, the tangent modulus $\left(E_{\mathrm{t}}\right)$ equal to $0.01 \mathrm{E}$ [21], and the material yield strength $\left(f_{y}\right)$ taken as $345 \mathrm{MPa}$, which was the standard value of one type of Chinese steel (i.e., Q345) widely used in engineering practice. It was noted that the material properties of brace was deemed the elastic component with an elasticity modulus equal to $206 \mathrm{Gpa}$ in order to get axial deformation of brace by elastic beam theory, which makes the acquisition of the connection deformation more accessibly (discussed in more detail in the later sections). It was also noted that the welds was assumed to be negligible in the FE model. The reasons were explained following: the welds can increase the bearing capacity due to increasing the thickness of the chord face which covered by the brace, but the welding process generate residual stresses which will reduce the strength of the tubular connections, and these distributions of residual stresses were difficult to estimated.

\subsection{Experimental test and $\mathrm{FE}$ analysis validation}

An experimental of CHS X-joint was carried out to validate the accuracy and rationality of the nonlinear FE technique in the Section 2.1. The specimen and its test displacement transducers arrangement was shown in Fig. 4, and the geometrical characteristics were listed in Table 1. The key material properties, i.e., yield strength $\left(f_{y}\right)$, ultimate strength $\left(f_{u}\right)$, elastic modulus $(E)$, and fracture elongation $(\zeta)$, obtained from tension coupon tests were summarized in Table 2.

Table 1 Average measured geometric properties

\begin{tabular}{|c|c|c|c|c|c|c|c|c|c|}
\hline Specimen & $D$ & $d$ & $T$ & $t$ & $\beta$ & $\gamma$ & $\tau$ & $\theta$ & $\varphi$ \\
\hline CHS-AX & 244.6 & 202.8 & 7.96 & 6.80 & 0.83 & 15.3 & 0.85 & 90 & 0 \\
\hline
\end{tabular}


Table 2 Average measured material properties

\begin{tabular}{lcccc}
\hline $\begin{array}{l}\text { Section } \\
(\mathrm{mm})\end{array}$ & $\begin{array}{l}f_{y} \\
(\mathrm{MPa})\end{array}$ & $\begin{array}{c}f_{u} \\
(\mathrm{GPa})\end{array}$ & $\begin{array}{c}\zeta \\
(\%)\end{array}$ \\
\hline $244.6 \times 7.96 \mathrm{CHS}$ & 375.3 & 545.1 & 209 & 32.7 \\
$202.8 \times 6.80 \mathrm{CHS}$ & 372.1 & 550.3 & 213 & 31.3 \\
\hline
\end{tabular}

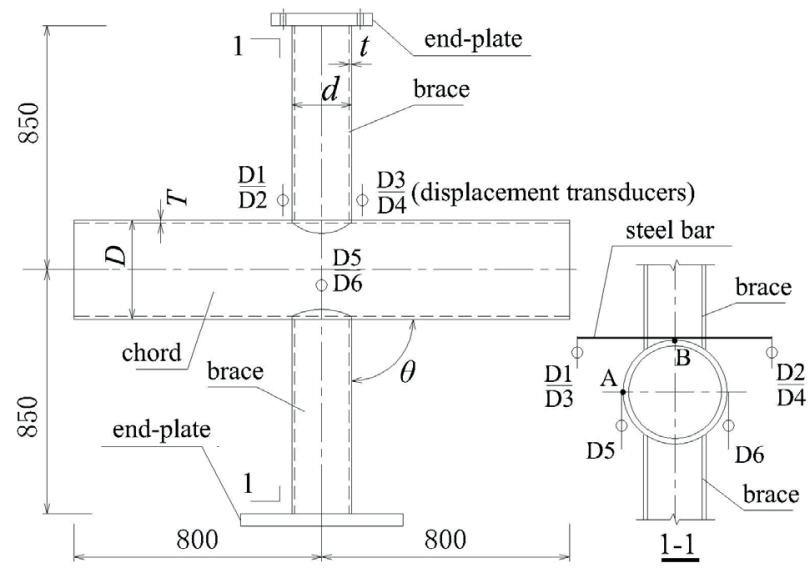

Fig. 4 Specimen and displacement transducers location

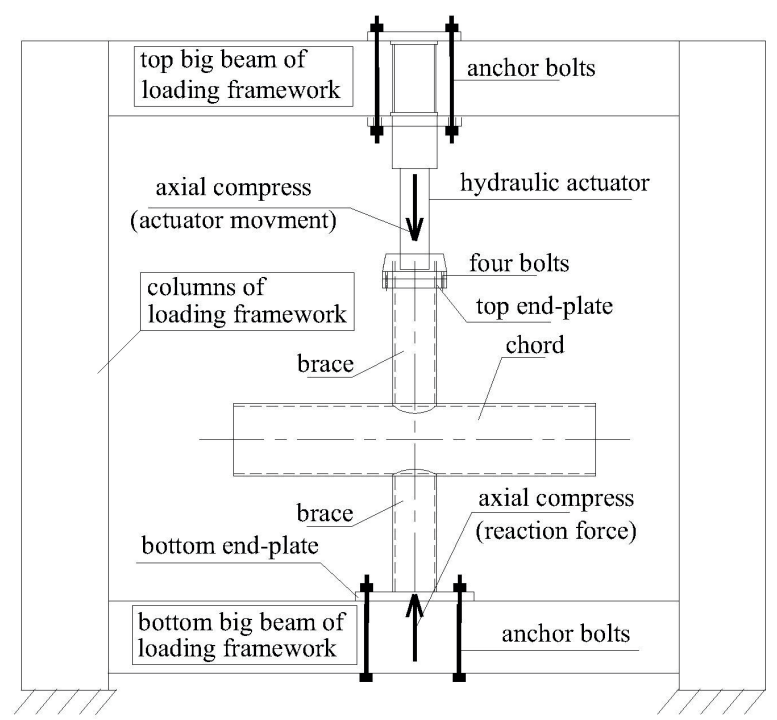

Fig. 5 Test setup and loading pattern

Fig. 5 shows the general arrangement for the CHS $\mathrm{X}$-joint test. The specimen was stand up, the chord placed horizontally and with both ends freely. One brace end was fixed to the bottom big beam of a laboratory loading frame through the bottom end-plate and four anchor bolts. Servo hydraulic actuator was connected to the other ends of the brace by the top end-plate and four high-strength bolts. The basic idea behind the details was to reduce the accessory device (i.e., both ends of the chord is freely), and the axial pressure at two brace ends can be applied synchronously through only one hydraulic actuator mounted to the top beam of the loading frame.
In the test, recognizable concave deformation was visually observed on the chord wall near the saddle region when the load (brace axial compression) was about $385 \mathrm{kN}$, then the concave deformation became more evident. The failure mode of the specimen demonstrated chord wall buckling near brace/chord intersection and was compared with the FE result, and the comparison of load-connection deformation responses ( $P-\delta$ curves) between the test results and FE results was also listed, as shown in Fig. 6. It was noted that there were two methods, direct and indirect methods, to obtain the connection deformation (i.e., $\delta$ ) for both experimental and FE simulation. As for direct method, proposed by Voth and Packer [22], the connection deformation was defined as the change in vertical distance between the center point at chord (point A in Fig. 4) and a point at the crown of the CHS chord (point B in Fig. 4), i.e., $\delta=\delta_{\mathrm{B}}-\delta_{\mathrm{A}}$. In the test, the displacement of point $\mathrm{B}$ and Point $\mathrm{A}, \delta_{\mathrm{B}}$ and $\delta_{\mathrm{A}}$, adopted $\left(\delta_{1}+\delta_{2}+\delta_{3}+\delta_{4}\right) / 4$ and $\left(\delta_{5}+\delta_{6}\right) / 2$ respectively; where $\delta_{1}-\delta_{6}$ are the displacement measured by the six transducers from D1 to D6 respectively (see Fig. 4). As for indirect method, the connection deformation was defined as the average of connection deformations from the up surface and the bottom surface of chord walls near to brace/chord intersection, expressed as $\delta=\left(\delta_{\text {tlp }}-\delta_{\mathrm{ub}}-\delta_{\mathrm{bb}}\right) / 2$; where, $\delta_{\mathrm{tlp}}$ is the total displacement at the loading end (i.e., the change in vertical distance between the two brace ends) and measured by the internal displacement transducer of the actuator, $\delta_{\text {ub }}$ and $\delta_{\mathrm{bb}}$ are the axial deformations of the up brace and the bottom brace calculated by the elastic bar theory.

It can be inferred from Fig. 6 that both failure mode and $P$ - $\delta$ curves of FE model correlates reasonably well with the experimental test results, and $P-\delta$ curves matching closely both the values and general response when $\delta<11 \mathrm{~mm}$ (about $4.5 \% \mathrm{D}$ ). Moreover, the $P$ - $\delta$ curves obtained by direct method and indirect method are also very close while $\delta<11 \mathrm{~mm}$. However, the six transducers ( $D 1$ to $D 6)$ is deviated the original location due to the excessive deformation of chord wall in the post-loading of the experiment, so the corresponding vertical displacement measured by the six transducers is wrong and deleted. The ultimate strength from test and FE are 425.5 and $419.9 \mathrm{kN}$, respectively, these two values are also very close to the predicted values of 379.5 and $397.7 \mathrm{kN}$ from the corresponding uniplanar CHS X-joints capacity formulae (under brace compression) in the EN 1993-1-8:2005 [18] and GB 50017-2017 [19], which expressed as following Eq. (1) and Eq. (2). Therefore, the FE modeling and 
analysis techniques in the Section 2.1 were thus deemed suitable for use as a numerical parametric analysis of the CHS X-joints.

$$
\begin{aligned}
& N_{\mathrm{EC} 3}=\frac{5.2 f_{y} T^{2}}{(1-0.81 \beta) \sin \theta}, \\
& N_{G B 50017}=\frac{5.45 f_{y} T^{2}}{(1-0.81 \beta) \sin \theta} .
\end{aligned}
$$

\section{Effects of out-of-plane brace-to-chord angle on the force transfer}

Based on the stress distribution of the brace near the brace/ chord intersection form FE results, the mechanical transmission analysis between the brace and chord can be carried out, the specific process was as follows. Firstly, a row of elements of the brace, closest to the intersection in the FE model, was numbered from 1 (the element near the up saddle point of $A_{1}$ ) to $n$ (the element near the bottom saddle point of $A_{2}$ ), as shown in Fig. 7(a). Then, obtained the $\sigma_{11}{ }^{\mathrm{j}}$ (the stress component along the brace axis) of each element by using the average of the stresses at two integration points (a S8R element have four integration points) close to the intersection of brace and chord, where superscripted $\mathrm{j}$ (from 1 to $\mathrm{n}$ ) indicated the $\mathrm{j}$-th element. Therefore, the
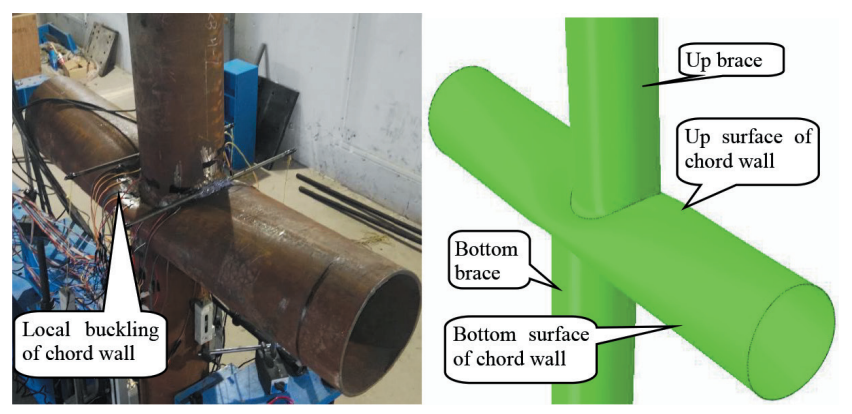

(a) Failure mode from test and FE

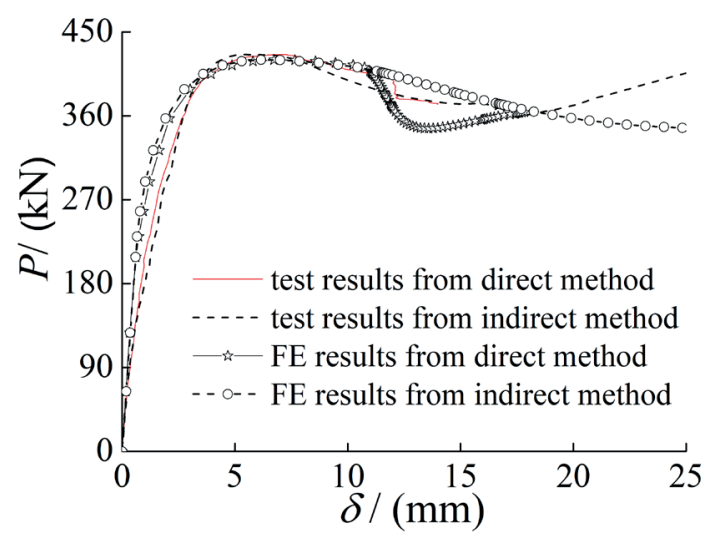

(b) load-connection deformation $(P-\delta)$ curves

Fig. 6 Comparison between test results and FE results elastic stress distribution along the intersection line was acquired, as highlighted in Fig. 7(b) for a typical engineering application of CHS X-joints with geometric parameters of $\beta=0.7, \varphi=0$ o and $20^{\circ}$. It can be found that the stress distribution characteristics of the uniplanar X-joint indicate that most load (brace axial compression) was transferred at the two saddles of the brace, which the same as the result of Togo [23]. However, $20^{\circ}$ of out-of-plane brace-to-chord angle (i.e., $\varphi$ ) can greatly changes the elastic stress distribution, most loads was transferred to near the up saddle point (point $A_{1}$ in Fig. 7(b)) of the brace but little load transferred near the bottom saddle point (point $\mathrm{A}_{2}$ in Fig. 7(b)).

Fig. 8 shows the elastic stress distribution comparing the CHS X-joints with different $\varphi$. Where, the geometric parameters of the $\mathrm{X}$-joints are $D=250 \mathrm{~mm}, \theta=90 \mathrm{o}, \gamma=10$, $\tau=0.8, \beta=0.4$ and 0.7 , and $\varphi=0^{\circ}, 5^{\circ}, 10^{\circ}, 20^{\circ}, 30^{\circ}$; the abscissa is the element number (the number is incremented from $A_{1}$ to $A_{2}$, see Fig. 7), and the ordinate is expressed in

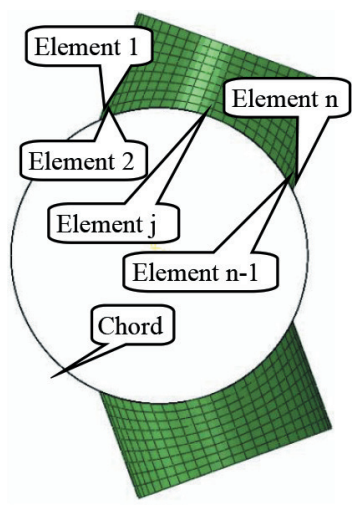

(a) Element number

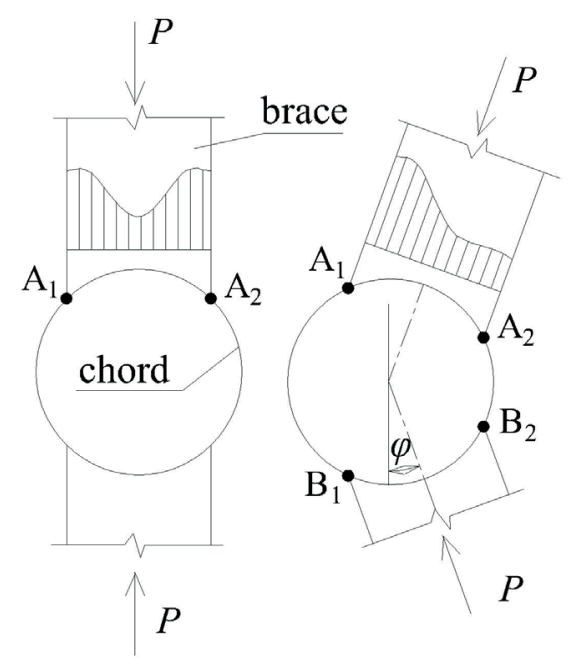

(b) Elastic stress distribution with $\varphi=0^{\circ}$ and $20^{\circ}$

Fig. 7 Element number of brace near to brace/chord intersection and elastic stress distribution comparison 


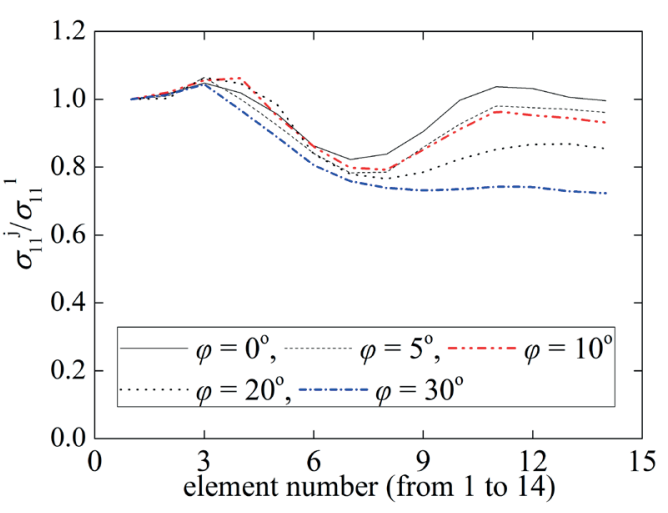

(a) CHS X-joint with $\beta=0.4$

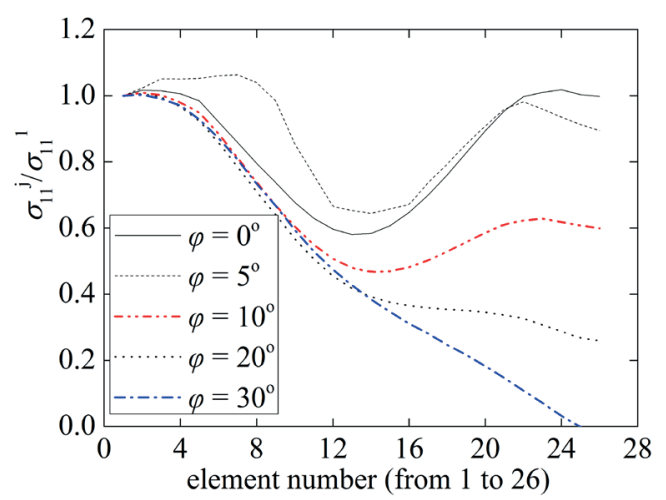

(b) CHS X-joint with $\beta=0.7$

Fig. 8 Effects of out-of-plane brace-to-chord angle $(\varphi)$ on the elastic stress distribution

terms of the normalized stress of $\sigma_{11}{ }^{\mathrm{j}} / \sigma_{11}{ }^{1}$ to permit a direct comparison between the stress of the element 1 (near the up saddle point of $\mathrm{A}_{1}$ ) and that of other element (e.g., element of j) in every X-joint. It can be found that the out-of-plane $\varphi$ causes the up saddle (near the point $\mathrm{A}_{1}$, see Fig. 7) to share more the load from brace axial force, while reduces the load shared by the bottom saddle (near the point $\mathrm{A}_{2}$, see Fig. 7) and the crown. Furthermore, the angle $\varphi$ has a greater influence on the stress distribution (force transfer pattern) of the $\mathrm{X}$-joints with relative larger $\beta(=0.7)$ than that of the X-joints with smaller $\beta(=0.4)$.

\section{Effects of out-of-plane brace-to-chord angle on the ultimate capacity}

Three groups CHS X-joints ( $\beta=0.4,0.7$ and 0.9$) \mathrm{FE}$ results are studied the effects of the angle $\varphi$ on the compression-connection deformation ( $\mathrm{P}-\delta$ ) curves, as shown in Fig. 9. Where, the deformation (i.e., $\delta$ ) was obtained by indirect method, the other parameters of the CHS X-joints are $\mathrm{D}=250 \mathrm{~mm}, f_{y}=345 \mathrm{MPa}, \theta=90^{\circ}, \gamma=10, \tau=0.8$; and the $\varphi$ is not great than $20^{\circ}$ when $\beta$ equal to 0.9 in order to avoid forming overlap CHS X-joint which behavior is quite different from gap $\mathrm{X}$-joint. It was found that the angle $\varphi$ has little effect on the ultimate capacity $\left(P_{\mathrm{u}}\right)$ of the $\mathrm{X}$-joints with small $\beta(=0.4)$, but has relative greater influence on the capacity of X-joints with medium or larger $\beta(=0.7,0.9)$, even if the angle $\varphi$ is tiny of $5^{\circ}$. Where, the capacity $P_{\mathrm{u}}$ adopted the first peak load for those X-joints with pronounced peak load in the $P$ - $\delta$ curves and the corresponding deformation $\delta_{\mathrm{fpl}}$ is less than $0.03 D$; otherwise, $P_{\mathrm{u}}$ adopted the load corresponding to $\delta=0.03 D$, a deformation limit criteria proposed by Lu et al. [24-31].

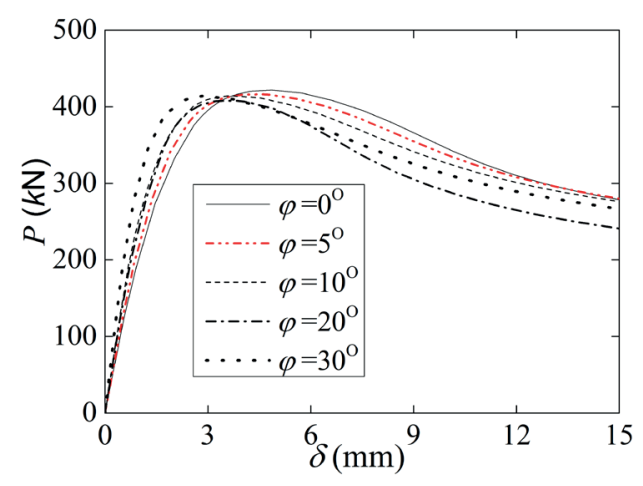

(a) CHS X-joint with $\beta=0.4$

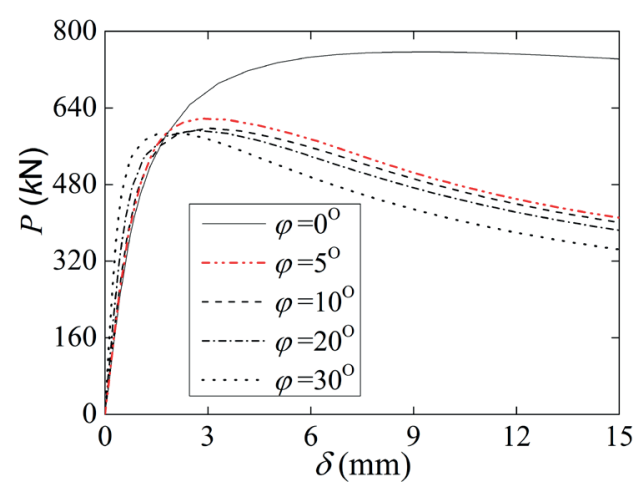

(b) CHS X-joint with $\beta=0.7$

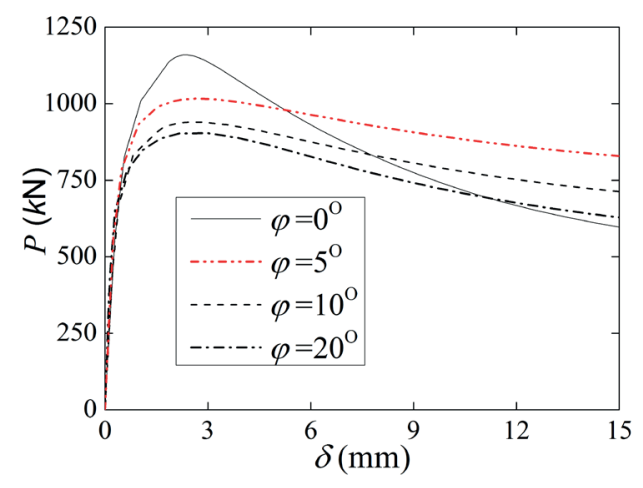

(c) CHS X-joint with $\beta=0.9$

Fig. 9 Effects of out-of-plane brace-to-chord angle $(\varphi)$ on the loadconnection deformation $(P-\delta)$ curves 
An extensive parametric analysis of $131 \mathrm{FE}$ models for both multiplanar and uniplanar CHS X-joints was carried out, in which $D=250 \mathrm{~mm}, \tau=0.8, f_{y}=345 \mathrm{Mpa}, \beta=0.4$, $0.7,0.9 ; \gamma=10,20,40 ; \theta=90^{\circ}, 75^{\circ}, 45^{\circ}, 30^{\circ}$, and $\varphi=0^{\circ}$, $10^{\circ}, 20^{\circ}, 30^{\circ}$. Then 99 data about $P_{\mathrm{u}}{ }^{\mathrm{mp}} / P_{\mathrm{u}}{ }^{\text {up }}$, the ratio of multiplanar CHS X-joints ultimate capacity $\left(P_{\mathrm{u}}^{\mathrm{mp}}\right)$ to that of the corresponding uniplanar $\mathrm{X}$-joints $\left(P_{\mathrm{u}}{ }^{\text {up }}\right)$ and defined as out-of-plane brace-to-chord angle (OPBCA) influence factor, were obtained and utilized to investigate the influence of the angle $\varphi$ on the capacity of the CHS X-joints, as shown in Fig. 10. It can be inferred form the Fig. 10 that almost all values of OPBCA influence factor are less than 1.0, indicating that the capacity of multiplanar $\mathrm{X}$-joints is lower than that of the corresponding uniplanar $\mathrm{X}$-joints, i.e., the angle $\varphi$ is unfavorable to the capacity of the CHS X-joints under brace compression. Furthermore, the $P \mathrm{u}^{\mathrm{mp}} / P_{\mathrm{u}}{ }^{\text {up }}$ of these CHS X-joints with $\beta=0.4$ or $\theta=30^{\circ}$ are mostly greater than 0.9 , some even exceed 0.95 ; while the $P_{\mathrm{u}}{ }^{\mathrm{mp}} / P_{\mathrm{u}}$ up of these $\mathrm{X}$-joints with $\beta \geq 0.75$ (0.75 and 0.9$)$ and $\theta \geq 75^{\circ}\left(75^{\circ}\right.$ and $\left.90^{\circ}\right)$ are almost less than 0.85 , some even less than 0.75 . Therefore, the influence of the angle $\varphi$ on the capacity of the later joints (with lager $\beta$ and $\theta$ ) is relative larger, because the discrepancy between the two load transfer region of $\mathrm{A}_{1} \mathrm{~B}_{1}$ (the distance between the up saddle points of the two braces, see Fig. 7(b)) and $\mathrm{A}_{2} \mathrm{~B}_{2}$ (the distance between the bottom saddle points of the two braces, see Fig. 7(b)) is increased with the increasing of $\beta$ and $\theta$, which lead to more difference between the multiplanar X-joints and the corresponding uniplanar X-joints.

Based on the capacity predict equations of brace compression loaded uniplanar X-joints in the current design specifications of EN 1993-1-8:2005 [18] or Chinese GB 50017-2017 [19], the capacity predict equations of the multiplanar X-joints was established by multiplying the OPBCA influence factor named $f(\varphi)$, which reflecting the influence of the angle $\varphi$ on the capacity. Then after analysis and multiple attempts on 99 data of $P_{\mathrm{u}}{ }^{\mathrm{mp}} / P_{\mathrm{u}}$ up from FE results, it was found that the item of ' $(1-\sin \varphi)^{\mathrm{g}(\beta, \sin \theta))^{\prime}}$ can be approximately fit the relationship between these $P_{\mathrm{u}}{ }^{\mathrm{mp}} / P_{\mathrm{u}}$ up ratios and the geometrical parameters of the CHS X-joints. Moreover, these item equal to 1.0 when $\varphi$ is $0^{\circ}$, i.e., translate into the uniplanar $\mathrm{X}$-joints. As for the $\mathrm{g}(\beta, \sin \theta)$, it can be adopted relatively simple and commonly used quadratic polynomial function, and the constant coefficients in the polynomial function can be determined based on the results of non-linear regression analysis. As a result, the equation for the OPBCA influence factor was established and expressed as Eq. (3): $f(\varphi)=(1-\sin \varphi)^{\left(0.85+1.66 \beta-4.83 \sin \theta-2.47 \beta^{2}+2.46(\sin \theta)^{2}+3.31 \beta \sin \theta\right)}$.

Where, $\beta$ is brace-to-chord diameter ratio, $\varphi$ and $\theta$ are out-of-plane and in-plane brace-to-chord angles, respectively. And based on the results listed in the Fig. 10, the additional condition of ' $0.72 \leq f(\varphi) \leq 1.0^{\prime}$ must also be met.

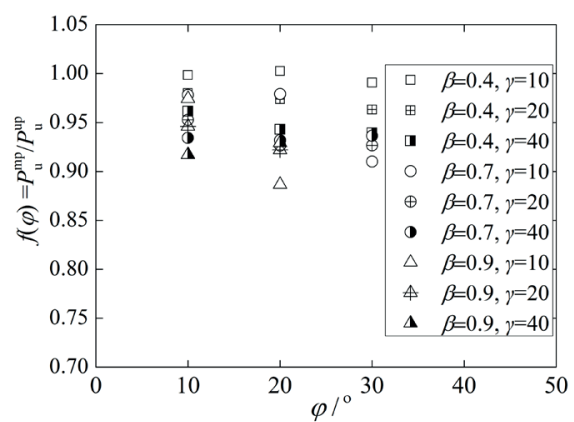

(a) CHS X-joint with $\theta=30^{\circ}$

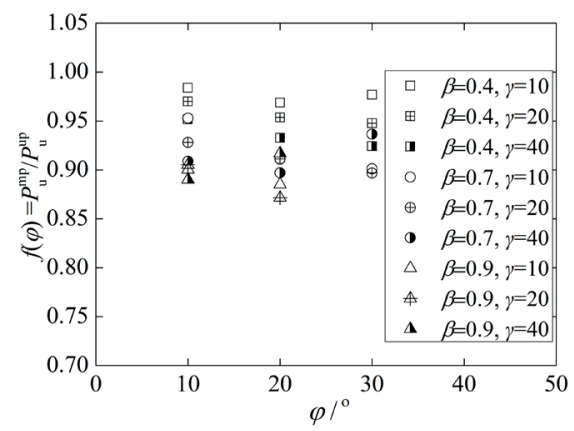

(b) CHS X-joint with $\theta=45^{\circ}$

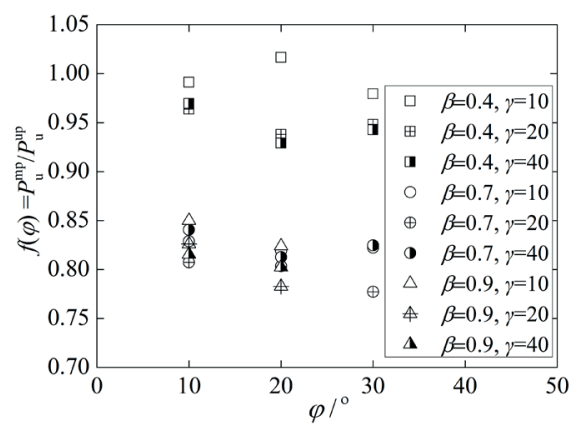

(c) CHS X-joint with $\theta=75^{\circ}$

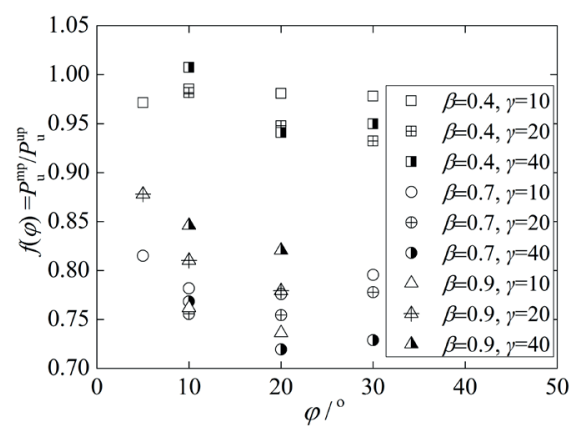

(d) CHS X-joint with $\theta=90^{\circ}$

Fig. 10 The influence coefficient of $f(\varphi)$ changed with the parameters $\theta, \varphi, \beta$ and $\gamma$ 
Table 3 Geometric properties for new 39 FE models of CHS X-joints

\begin{tabular}{|c|c|c|c|c|c|c|c|c|c|c|c|}
\hline$\beta$ & 0.5 & 0.5 & 0.5 & 0.6 & 0.6 & 0.6 & 0.7 & 0.8 & 0.8 & 0.8 & 0.8 \\
\hline$\gamma$ & 10 & 10 & 30 & 10 & 10 & 25 & 10 & 15 & 30 & 15 & 30 \\
\hline$\tau$ & 0.8 & 0.8 & 0.8 & 0.8 & 0.6 & 0.8 & 0.8 & 0.8 & 0.8 & 0.8 & 0.8 \\
\hline$\theta$ & 90 & 45 & 60 & 90 & 45 & 55 & 60 & 50 & 50 & 80 & 80 \\
\hline$\varphi$ & $\begin{array}{c}0,10, \\
20,30\end{array}$ & $\begin{array}{c}0,10, \\
20,30\end{array}$ & $\begin{array}{c}0,15, \\
25,35\end{array}$ & $\begin{array}{c}0,10 \\
20,30\end{array}$ & $\begin{array}{c}0,10, \\
20,30\end{array}$ & $\begin{array}{c}0,18 \\
32\end{array}$ & $\begin{array}{c}0,10 \\
20,30\end{array}$ & $\begin{array}{c}0,13 \\
25\end{array}$ & $\begin{array}{c}0,13 \\
25\end{array}$ & $\begin{array}{c}0,13 \\
25\end{array}$ & $\begin{array}{c}0,13 \\
25\end{array}$ \\
\hline
\end{tabular}

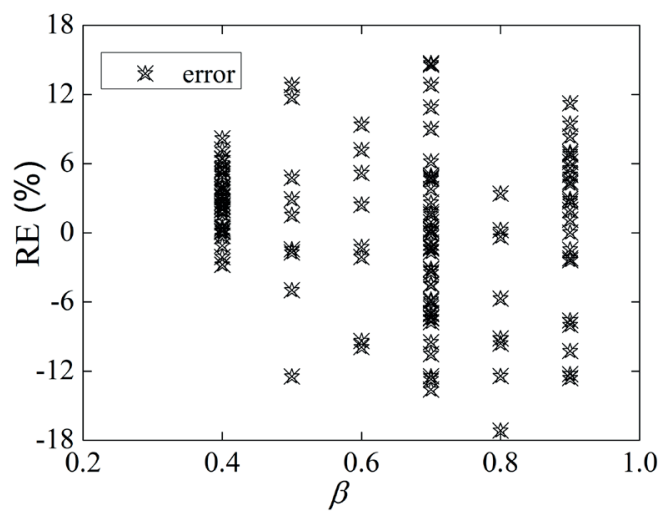

Fig. 11 Errors of $f(\varphi)$ between calculated results and FE results

There were total 127 data from FE results, including the above 99 data and new 28 data from 39 FE model of the CHS X-joints with parameters listed in Table 3 $\left(D=250 \mathrm{~mm}, f_{y}=345 \mathrm{Mpa}\right)$, were utilized to evaluate fitting results of the factor $f(\varphi)$, as shown in Fig. 11. Where, abscissa and ordinate are geometric parameters $\beta$ and relative errors (RE) between FE results and predicted results of Eq. (3), respectively. It can be inferred that most of RE are within $10 \%$, and the maximum value of RE is about $17 \%$. Therefore, Eq. (3) can well reflect the influence of the brace-to-chord out-of- plane angle (i.e., $\varphi$ ) on the capacity of the CHS X-joints under brace compression.

\section{Conclusions}

An experimental of CHS X-joint was carried out to validate the rationality of FE model. Then an extensive FE parameters analyses were carried out to study the static behavior difference between multiplanar CHS X-joints and the corresponding uniplanar CHS X-joints under brace compression. Finally, the effects of out-of-plane brace-to-chord angle (OPBCA) on the CHS X-joint were researched, so, the following conclusions can be made.

1. The failure modes, load-connection deformation curves and ultimate capacity of FE model correlates reasonably well with the $\mathrm{CHS} \mathrm{X}$-joint experimental tests results.
2. OPBCA causes the up saddle to bearing more load transferred from brace axial force, and reduces the load at bottom saddle, while the loads at the two saddles are the same to the uniplanar X-joints. Moreover, the angle $\varphi$ has more influences on the mechanical transmission of the X-joints, and compared with smaller $\beta$, larger $\beta$ is more likely to affect its mechanical transmission.

3. OPBCA is unfavorable to the capacity of the CHS $\mathrm{X}$-joints under brace compression, especially the joints with relative large $\beta(=0.75$ and 0.9$)$ and in-plane angle $\theta\left(=75^{\circ}\right.$ and $\left.90^{\circ}\right)$. Furthermore, an equation considering the OPBCA influence factor is established, which takes into account the adverse effects of OPBCA and can extend the capacity prediction formulae of uniplanar X-joints to the multiplanar CHS $\mathrm{X}$-joints. Especially, the equation with the OPBCA influence factor has been validated favorably.

\section{Acknowledgments}

The financial support from the National Natural Science Foundation of China (No.51778538), the China Scholarship Council (No. 201708330384, No.201707005100), and the China-Indonesia Joint Research Center for High-speed Railway Technology (No.KY201801005) are acknowledged and sincerely appreciated by the authors. 


\section{References}

[1] Liu, C., Li, Q., Lu, Z., Wu, H. "A review of the diagrid structural system for tall buildings", The Structural Design of Tall and Special Buildings, 27(4), 2018.

https://doi.org/10.1002/tal.1445

[2] Liu, C., Ma, K. "Calculation model of the lateral stiffness of highrise diagrid tube structures based on the modular method", The Structural Design Tall and Special Buildings, 26(4), 2017. https://doi.org/10.1002/tal.1333

[3] Gerdeen, J. C. "A critical evaluation of plastic behavior data and a united definition of plastic loads for pressure components", 1st ed., Welding Research Council, New York, NY, USA, 1979.

[4] Choo, Y. S., Qian, X. D., Liew, J. Y. R., Wardenier, J. "Static strength of thick-walled CHS X-joints - Part I. New approach in strength definition", Journal of Constructional Steel Research, 59(10), pp. 1201-1228, 2003.

https://doi.org/10.1016/S0143-974X(03)00054-3

[5] Choo, Y. S., Qian, X. D., Liew, J. Y. R., Wardenier, J. "Static strength of thick-walled CHS X-joints - Part II. Effect of chord stresses", Journal of Constructional Steel Research, 59(10), pp. 1229-1250, 2003. https://doi.org/10.1016/S0143-974X(03)00053-1

[6] Fung, T. C., Soh, C. K., Gho, W. M., Qin, F. "Ultimate capacity of completely overlapped tubular joints: I. An experimental investigation", Journal of Constructional Steel Research, 57(8), pp. 855880, 2001.

https://doi.org/10.1016/S0143-974X(01)00013-X

[7] Fung, T. C., Soh, C. K., Gho W. M. "Ultimate capacity of completely overlapped tubular joints. II: Behavioral study", Journal of Constructional Steel Research, 57(8), pp. 881-906, 2001. https://doi.org/10.1016/S0143-974X(01)00014-1

[8] Feng, R., Young, B. "Theoretical analysis of cold-formed stainless steel tubular joints", Engineering Structures, 83, pp. 99-115, 2015. https://doi.org/10.1016/j.engstruct.2014.10.030

[9] Iskander, M. S., Shaat, A. A., Sayed-Ahmed E. Y., Soliman, E. A. "Strengthening CHS T-joints subjected to brace axial compression using through-bolts", Journal of Constructional Steel Research, 128, pp. 555-566, 2017.

https://doi.org/10.1016/j.jcsr.2016.09.019

[10] Zhu, L., Han, S., Song, Q., Ma, L., Wei, Y., Li, S. "Experimental study of the axial compressive strength of CHS T-joints reinforced with external stiffening rings", Thin-Walled Structures, 98(B), pp. 245-251, 2016.

https://doi.org/10.1016/j.tws.2015.09.029

[11] Zhu, L., Yang, K., Bai, Y., Sun, H., Wang, M. "Capacity of steel CHS $\mathrm{X}$-joints strengthened with external stiffening ring in compression", Thin-Walled Structures, 115, pp. 110-118, 2017. https://doi.org/10.1016/j.tws.2017.02.013

[12] Fu, Y., Tong, L., He, L., Zhao, X.-L. "Experimental and numerical investigation on behavior of CFRP-strengthened circular hollow section gap K-joints", Thin-Walled Structures, 102, pp. 80-97, 2016. https://doi.org/10.1016/j.tws.2016.01.020
[13] Zhao, B., Liu, C., Yan, Z., Chen, X., Cai, Y. "Semi-rigidity Connection Model for Unstifened CHS X-type Joints Subjected Out-of-plane Bending", International Journal of Steel Structures, 19(3), pp. 834-849, 2019.

https://doi.org/10.1007/s13296-018-0168-X

[14] Chen, Y., Feng, R., Xiong, L. "Experimental and numerical investigations on double-skin CHS tubular X-joints under axial compression", Thin-Walled Structures, 106, pp. 268-283, 2016. https://doi.org/10.1016/j.tws.2016.05.007

[15] Nassiraei, H., Lotfollahi-Yaghin, M. A., Ahmadi, H. "Static performance of doubler plate reinforced tubular T/Y-subjected to brace tension", Thin-Walled Structures, 108, pp. 138-152, 2016. https://doi.org/10.1016/j.tws.2016.08.012

[16] Liu, C., Ma, K., Wei, X., He, G., Shi, W., Zhou, Y. "Shaking Table Test and Time-history Analysis of High-rise Diagrid Tube Structure", Periodica Polytechnica Civil Engineering, 61(2), pp. 300-312, 2017.

https://doi.org/10.3311/PPci.9243

[17] Lan, X., Wang, F., Ning, C., Xu, X., Pan, X., Luo, Z. "Strength of internally ring-stiffened tubular DT-joints subjected to brace axial loading", Journal of Constructional Steel Research, 125, pp. 88-94, 2016. https://doi.org/10.1016/j.jcsr.2016.06.012

[18] CEN "EN 1993-1-8:2005 Eurocode 3: Design of Steel StructuresPart 1-8: Design of Joints", European Committee for Standardization, Brussels, Belgium, 2005.

[19] MOHURD "GB 50017-2017 Standard for Design of Steel Structures", Ministry of Housing and Urban-Rural Development, Beijing, China, 2018. (in Chinese)

[20] Abaqus "Abaqus Analysis User's Manual", [online] Available at: http://130.149.89.49:2080/v6.7/books/usb/default.htm [Accessed: 29 May 2019]

[21] BSI "BS EN 1993-1-5:2006 Eurocode 3. Design of steel structures, Part 1-5: Plated structural elements", British Standards Institution, London, UK, 2006.

[22] Voth, A. P., Packer, J. A. "Numerical study and design of skewed X-type branch plate-to-circular hollow section connections", Journal of Constructional Steel Research, 68(1), pp. 1-10, 2012. https://doi.org/10.1016/j.jcsr.2011.06.005

[23] Togo, T. "Experimental Study on Mechanical Behavior of Tubular Joints", Osaka University, 1967.

[24] Lu, L. H., De Winkel, G. D., Yu, Y., Wardenier, J. "Deformation limit for the ultimate strength of hollow section joints", Tubular Structures VI: Proceedings Sixth International Symposium on Tubular Structures, Melbourne, Australia, 1994, pp. 341-347.

[25] Liu, C., Ni, X., Wu, H., Wei, X., He, B. "Calculation Theory and Test Verification for Skeleton Curve of T-shaped Shear Walls", Structural Engineering International, 27(2), pp. 281-291, 2017. https://doi.org/10.2749/101686616X1311

[26] Qiu, W., Lu, F., Wang, G., Huang, G., Zhang, H., Zhang, Z., Gong, C. "Evaluation of mechanical performance and optimization design for lattice girders", Tunnelling and Underground Space Technology, 87, pp. 100-111, 2019. https://doi.org/10.1016/j.tust.2019.02.008 
[27] Liu, C., Wei, X., Wu, H., Li, Q., Ni, X. "Research on Shear Lag Effect of T-shaped Short-leg Shear Wall", Periodica Polytechnica Civil Engineering, 61(3), pp. 602-610, 2017.

https://doi.org/10.3311/PPci.9491

[28] Zhao, B., Liu, C., Wu, H., Ge, Y., Yang, J., Yi, Q. "Study on outof-plane flexural stiffness of unstiffened multi-planar CHS X-joints", Engineering Structures, 188, pp. 137-146, 2019. https://doi.org/10.1016/j.engstruct.2019.03.023

[29] Zhou, N., Shi, W., Shang, J. "Seismic response of a light steel structure integrated building with steel mortise-tenon connections", Advances in Structural Engineering, 22(5), pp. 1225-1237, 2019. https://doi.org/10.1177/1369433218811533
[30] Liu, X., Sheng, K., Li, Z., Gan, L., Shan, H., Hong, B. "Experimental Research on Foamed Mixture Lightweight Soil Mixed with Fly-Ash and Quicklime as Backfill Material behind Abutments of Expressway Bridge", Advances in Materials Science and Engineering, 2017. https://doi.org/10.1155/2017/5767103

[31] Zhao, B. D., Chen, Y., Liu, C. Q., Wu, H. D., Wang, T., Wei, X. D. "An axial semi-rigid connection model for cross-type transverse branch plate-to-CHS joints", Engineering Structures, 181, pp. 413426, 2019

https://doi.org/10.1016/j.engstruct.2018.12.042 